\title{
Dublex Doppler US Diagnosis and Follow Up of a Post Traumatic Pseudoaneurysm of the Radial Artery
}

\author{
Besa Hidri $^{1}$, Durim Çela ${ }^{2}$, Diamant Shtiza ${ }^{3}$ \\ ${ }^{1 .}$ Service of Pediatric Imaging. University Hospital Center "Mother Teresa" Tirana, Albania \\ ${ }^{2}$ Service of Imaging. University Hospital Center "Mother Teresa" Tirana, Albania \\ ${ }^{3}$ Service of Pediatric. University Hospital Center "Mother Teresa" Tirana, Albania
}

\begin{abstract}
Introduction: Post traumatic pseudoaneurysms of the radial artery are rare complications in children. They are seen mostly later; weeks or even months after a penetraiting trauma injury. The serious complications of radial artery pseudoaneurysm, such as spontaneous rupture, ischemia and compartment syndrome emerge early diagnosis and treatment. A high index of suspicion is needed for clinicians to avoid confusion with an abscess or hematoma. Duplex ultrasound is the first method to establish the diagnosis of a pseudoaneurism. It is now widely accepted as the the modality of choice for diagnosis of pseudoaneurism of extremities. Early detection and therapeutic intervention before the pseudoaneurysm manifests clinically with complications is very important. Timely adequate diagnosis and treatment has decrease the morbidity and mortality rates for pseudoaneurysms. As a non invasive method it is now widely used olso as a guided therapeutic option before and instead surgical treatment. Case report: In this article we present a case of a post traumatic pseudoaneurysm in the radial artery of a 12 years old girl diagnosed by dublex ultrasound. The pseudoaneurism was detected 2 weeks after a sharp injury caused by a piece of glass at the medial distal part of the right forearm. The pseudoaneurysm was unfortunately first confused as an abscess in gray scale ultrasound. Follow up dublex US for 2 weeks without treatment revealed thrombosed of the pseudoaneurism continuing with recanalisation of blood flow from radial artery to pseudoaneurism. Conclusion: Imaging with dublex color doppler resolve the diagnosis, otherwise the confusion with an abscess, as in our patient may have the disastrous consequence of incision and drainage. Duplex ultrasound is recommended as an efficiency diagnostic method for forearm and wrist swelling in children after injury. Timely adequate diagnosis and treatment has decrease the morbidity and mortality rates for pseudoaneurysms. This case remind us for careful examination and follow up of the swelling mass after trauma in children. In these cases dublex color Doppler is an imperative investigation to avoid confusion with an abscess or hematoma.
\end{abstract}

Keywords: Dublex doppler ultrasound, pseudoaneurism, radial artery, children.

\section{Introduction}

A pseudo-aneurysm, olso known as false aneurysm is a collection of blood leaking from a damage artery, with communication between them. This passage of flow from the originating artery to pseudoaneurysm is called the neck. It is an important key finding for differentiation between a true aneurism and false aneurism. The perfused collection wall is composed from surrounding tissue or by the media or adventitia.

Pseudoaneurysms are a rare complication of radial arterial injury especially in children [4]-[21]-[13].The arterial lesions are not detected usually at the time of injury and present later with pain and swelling at the site of the original injury.

Complications such as thromboembolism, and infection, local compression on neurovascular structures or rupture out to the surrounding tissue emerge the immediate treatment. The poor support of the aneurysm wall is the cause of the rupture. Vascular insufficiency from thromboembolism, is an important clinical sign for early diagnosis and surgical management.

The common causes of radial pseudo aneurysm in children are penetrating trauma and iatrogenic arterial injury [11].

Post traumatic pseudoaneurysms are usually caused from knife or glass puctures of the artery. Jatrogenic pseudoaneurysms are the result of percutaneous surgical procedures or the use of an artery for injections [10].

Patients with radial artery pseudoaneurysms can present late, in weeks or even months after the injury with pain and swelling of the wrist. The differential diagnoses is with abscess, haematoma, or true aneurysm. Pseudoaneurysms may clinically mistaken with them.

Colour dublex doppler ultrasound is the primary investigation of choise for diagnosis a pseudoaneurism [10].

\section{Case Report}

A 12 year-old girl was admitted in our service for ultrasound examination. She had a history of trauma before 2 weeks to her distal medial part of the right forearm. She had an accident with open injury on the antero medial part over the wrist. There was much bleeding at the time of accident and a pressure dressing was applied over the injured area. The parents send the girl immediately to the local city hospital. After removing the pressure dressing there was pulsatile blood at the area of injury. The emergency intervention was performed by a general surgeon who ligated the wound.

Two weeks later she noticed a smooth swelling mass over the ligated area of the injury. A rutine gray scale ultrasound 


\section{International Journal of Science and Research (IJSR) \\ ISSN (Online): 2319-7064 \\ Index Copernicus Value (2013): 6.14 | Impact Factor (2014): 5.611}

examination was performed at the local hospital. The diagnosis was suspicious for abscess and the girl was referred to the surgeon for incision and drainage. After consultation in our hospital the surgeon referred her again for another expertise ultrasound to our service.

Physical examination before ultrasound showed a soft swelling mass mith no palpable thrill cranial to the injury scar. She was afebrile and did not complain for pain, diminished sensacion or motor function. The forearm and the hand were well perfused (Fig 1).

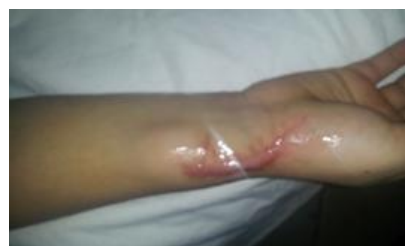

Figure 1: Physical examination showed a soft swelling mass mith no palpable thrill cranial to the injury scar of right distal forearm

Gray scale ultrasound examination realized in our service with a $12 \mathrm{MHz}$ linear probe ( US equipment Toshiba 6000) showed the presence of a round hypo-anechoic sacular subcutaneous structure with posterior enhancemet, localized cranial to the suture. The lesion was immediately deep to the skin surface not affecting underlying tendons, musculature, or bone. It had measurements $3 \times 2.5 \mathrm{~cm}$, with echogenic wall thickness of $3 \mathrm{~mm}$.

A turbulent echogenic flow was seen flowing inside from the base of the lesion, resembling of a aneurism (Fig 2).

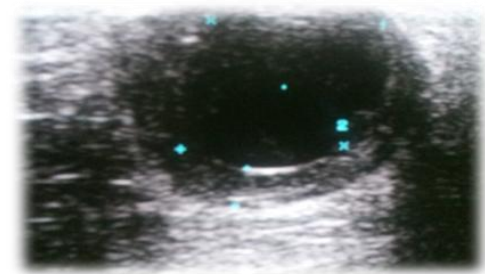

Figure 2: Gray scale US of the hypo-anechoic lesion with thicked wall and echogenic turbulent content inside

Color doppler ultrasound revealed intrasacular - turnover "red and blue" coming from radial artery. The communicated breech or the tubular neck between the radial artery and the sacular lesion was clearly seen in a length $5 \mathrm{~mm}$ with flow coming from radial artery to the aneurismal sac (Fig 3).

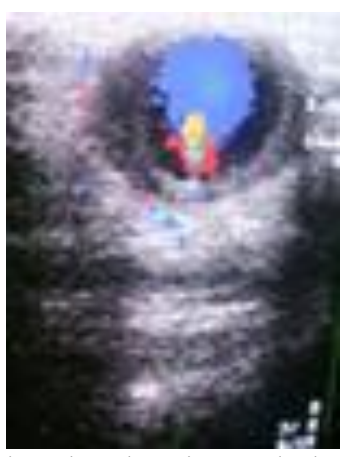

Figure 3: Color doppler showing turbulent flow inside the sac.
Dublex spectral doppler US showed a - to and - fro spectral waveform: representing flow during sistole and diastole (Fig. 4).

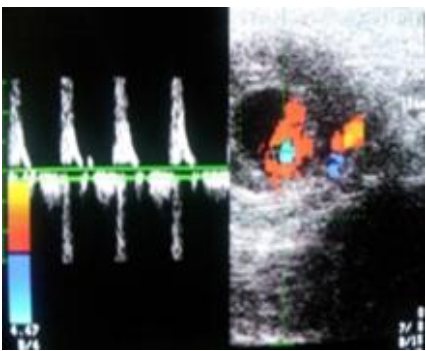

Figure 4: Dublex US showing bidirecsional arterial turbulent flow of pseudoaneurism.

The Dublex dopler US findings were all consistent with pseudoaneurism of radial artery.

The patient underwent olso a CT with IV angiogram which confirmed the presence of the pseudoaneurism connected with radial artery. She was follow up under close observation, because the parents wanted to send her in a specialized vascular clinic in Germany.

In follow up: after 2 days it was seen echogenic deposition under the wall consistent of clot formation, and less flow was seen in color Doppler inside the pseudoaneurism (Fig 5).

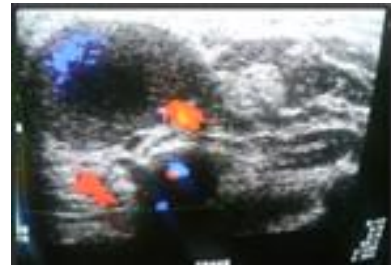

Figure 5: Echogenic deposition under the wall of pseudoaneurism. Reduced blood flow inside on color doppler.

At the third controll after 4 days from dhe second controll: gray scale ultrasoud showed homogene echogenic content representing clotts inside and color doppler showing no flow inside. These findings were consistent of thrombosed pseudoaneurism (Fig 6). The parents were happy with this natural favorable outcome.

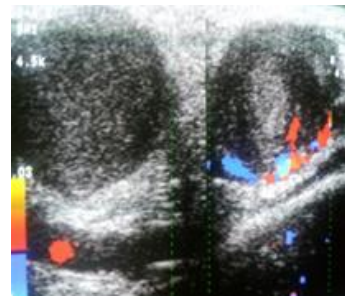

Figure 6: Thrombosed pseudoaneurism after 6 days observation without treatment.

Unfortunately after one week they come again with the girl for follow up. Dopler ultrasound revealed no thrombosis and remarcable flow inside the sac. These findings were consistent of recanalisation of blood flow from radial artery to pseudoaneurism. (Fig7(a),(b).) 


\section{International Journal of Science and Research (IJSR) \\ ISSN (Online): 2319-7064}

Index Copernicus Value (2013): 6.14 | Impact Factor (2014): 5.611

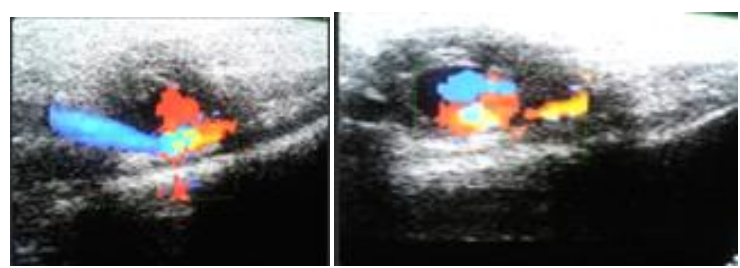

Figure 7: (a),(b). Recanalisation of blood flow from radial artery to pseudoaneurism.

Two weeks follow up without treatment, dublex ultrasound revealed a nonfavorable prognosis of this case of pseudoaneurism. The surgeon in a specialised vascular clinic in Germany performed resection of the pseudoaneurysm with restoration of arterial continuity by a venous interposition graft . There was full recovery of the pseudoaneurism without any consequenece of vascular perfusion and sensormotor disfunction of the patient's right hand.

\section{Discussion}

Pseudoaneurysm of an artery occurs as the result of a partial or full thickness traumatic wall disruption. As the result of the vessel wall injury the blood escape from the artery to the surrounding tissues. The created hematoma which is pulsatile is gradually surrounded by a fibrous wall capsule. The wall of the pseudoaneurism has no intima layer. Over time the blood can clott gradually within the fibrous wall. The difference of a pseudoaneurism from true aneurism is the composition of the wall. The true aneurysm wall is composed by 3 layers: the intima, media, or adventitia, whereas a false aneurism lack at least the intima.

The most common causes of radial pseudo aneurysm in children and adolescents are penetrating trauma and iatrogenic arterial injuries [11].

The main causes of pseudoaneurisms of extremities are as a consequence of coronary artery catheterization, arteriovenous shunting for dialysis, placement of indwelling catheterization, and arterial puncture for blood gas analysis [10].

The most common cause of jatrogenic pseudoaneurysms in children, is arterial catheterization, and the frequently involved vessel is the femoral artery. Post traumatic artery damage of extremities in children and infants is reported as the concequence venipuncture [6],[19].

Post-traumatic pseudoaneurysms happen rare in the peripheral arteries, especially in upper extremities and is generally a late sequela of artery wall injury. The most common causes of radial pseudo aneurysm in children are penetrating trauma and iatrogenic arterial injuries [13],[1]. The children with penetrating injuries of sharp cuts are more prone to pseudoaneurisms [4].

Many of these lesions are missed at the time of injury. They occur later with unknown mechanism, and present even months to years after the initial injury [20]-[13]-[1]. Posttraumatic pseudoaneurysms happen in every damaged artery after trauma, although femoral and radial arteries are the most common vessels.
There are no statistical data till now to the incidence of pseudoaneurysms in children. This is because of the rarity , and mostly reported as case presentation.

The clinical diagnosis is supported from the history of a trauma and physical examination. Most of post-traumatic pseudoaneurysms are the result of penetrating sharp injuries. The time of presentation, does'nt correlate exactly with the time of the injury. Sometimes, patients are admitted to hospitals with pseudoaneurysms weeks, months or even years after the trauma [4],[13].

Generally, the patients complain of a pulsatile mass or local swelling as in our case [2]. The history of a previous trauma makes it suspicious for hematoma or an abscess. Erythema, pain or local paresthesia may be other complains [13]. The physician should examine the patient carefully with digital examination, because pulsatility is an important clinical finding suggestive of pseudoaneurism.

Hemorrhagie from rupture is the most serious complication with life-threating consecuence. Vascular compromise may lead to ischemia of the surrounding tissues.

Thromboembolism as a potential complicationin, particularly in distal locations of extremities can lead to serious gangrene and amputation of the extremity or finger.

Neurologic symptoms secondary to nerve compression may be other common complications. Pseudoaneurism may undergo olso infection. Pseudoaneurisms with possible complications pose special attention for children in the aspect of growth discrepancy secondary to arterial ischemia [13],[16].

US is an accurate diagnostic tool for diagnosis post traumatic pseudoaneurysms. It is an accessible, portable, noninvasive method with high sensitivity and a specificity for detection of pseudoaneurysms. It is the method of choise for evaluation of volar wrist swelling [2].

A pseudoaneurysm in gray-scale US appears as a a hypoechoic cystic structure nearby a supplying artery. Ultrasound is practically used to differentiate between solid and cystic lesions adjacent to the radial artery in the wrist swollen area [15].

Pseudoaneurysms may be single or complex septated sac. Septa and occasionally concentric layers of hematoma may also be seen within the pseudoaneurysm

Grayscale ultrasound can be used to assess some of the features like the size, the number of compartments in the pseudoaneurysm, the length and width of the neck conection to the artery.

However, gray scale US is not diagnostic, since these findings are seen in a number of conditions, and may be confused with other simple or complex cystic lesions like hematomas or abscess [16].

Color Doppler US helps establish the diagnosis. Color Doppler is used in any anechoic-hypoechoic collection sac to 


\section{International Journal of Science and Research (IJSR) \\ ISSN (Online): 2319-7064}

Index Copernicus Value (2013): 6.14 | Impact Factor (2014): 5.611

detect or exlude flow inside. A pseudoaneurysm appears as a hypoechoic sac with colour flow present inside. It is seen tipically adjacent to the affected artery. Turbulent blood flow is seen by mixed coloring appearance, either red or blue. Dublex Ultrasound is essencial to differentiate pseudoaneurysm from non-communicating hematoma [15].

Blood flow within a psedoaneurism like in every cystic structure is characterized by a typical swirling motion called the "yin-yang sign". This tipical finding of flow may also be seen in saccular aneurysms.

Differential diagnosis is necessary in case of pseudoaneurysm because diagnosis made on this finding alone may not be sufficient and accurate. The key to confirm the diagnosis is visualization of a communicating neck between the sac and the feeding artery. In Dublex Doppler US is seen a typical "to-and-fro" waveform inside [11].

The "to" wave component represents blood entering the pseudoaneurysm during systole; the "fro" wave component represents blood exiting the pseudoaneurysm during diastole. Pulsed Doppler spectral analysis of the radial artery pseudoaneurysm has usually both patterns "yin-yang" and "to-and-fro" waveform. In follow up a spectral waveform analysis of the affected artery is useful for comparison [10]

The adjacent vein should be investigated to exclude the presence of thrombus [10].

The typical findings of pseudoaneurysm in triplex ultrasound are presence of a hypoechoic collection with pulsatility and turbulent flow inside.

Two hallmarks diagnosis of pseudoaneurysm are detection of the neck communication with the injured artery and "to and fro" spectral waveform pattern [10]. Color Doppler shows the characteristic yin-yang sign, whereas pulsed Doppler shows "to and fro" pattern.

Another advantage of dublex US is that it can be performed easily near the bed of the very ill patients, as a guide during thrombin injection or follow up after to ensure a completely pseudoaneurism [3]-[12]-[8].

In addition, it is very important to analise the US findings in the clinical context. In our case, the history of previous trauma allowed a more accurate diagnosis of the pseudoaneurysm of radial artery. US examination is olso required to access pseudoaneurysm before US guided intervention. Color Doppler US is accurate, noninvasive technique, and easy available.

CT Angiography is very good diagnostic method for pseudoaneurism, demonstrating a contrast filled sac arising from the donor artery [10]. Three-dimensional CT angiography allows visualization of the lesion from all angles, which is not possible with angiography. It is useful in cases where duplex ultrasound findings are equivocal. The radiation exposure and iodinated contrast agents risk makes it no suitable diagnostic tool particularly for children
Helical CT arteriography has been reported as the initial diagnostic method in most adults patients focal arterial injuries of upper extremities [18].

Conventional Angiography has been the standard method for the diagnosis of pseudoaneurysms in many years. It is important specially for surgeons in treatment planning. The disadvantages of angiography such as invasive nature, radiation and iodinated contrast material, makes it no suitable for children. Management of pseudoaneurisms is not unique. Treatment depends on the size and location of the aneurysm. For very small, stable, and asymptomatic pseudoaneurysms, it is recommended conservative treatment with follow up imaging after to see if spontaneous thrombosis has occurred [10] .

The conservative treatment includes clinical observation through watchful waiting and compression bandaging. Both are accessible and practical methods in treating peripheral pseudoaneurysms in children [5].

Observation has been recommended for asymptomatic smallpseudoaneurysms of arteries of only minor importance

In recent years, minimal invasive treatment methods using Dublex US have been applied in many cases. These methods included ultrasound-guided compression and percutaneous ultrasound-guided thrombin injection [11].

The positive results of ultrasound-guided injection of thrombin into radial artery pseudoaneurysm has shown this treatment method as a feasible alternative to surgical intervention [8].

These new treatments are not totally safety because of eventually complications such as thrombosis of the affected artery or peripheral vessels in case of thrombin injection. The more sensitive areas, are the head or hand [14].

Despite aforementioned treatment methods, surgical treatment remain standart for children. It includes: resection of the pseudoaneurysm with restoration of arterial continuity by an end-to-end anastomosis and reconstruction using a venous interposition graft as in our case.

Follow up with Dublex US evaluates the anastomotic patency to ensure optimal circulation in the limb, which is during the growth, in the pediatric population [17].

\section{Conclusion}

Post-traumatic pseudoaneurym of radial artery is a potentially serious complication in children. A swelling mass in the forearm or wrist in the context of previous injury should raise the suspicious of pseudoaneurism.

Dublex ultrasound establish a rapid and accurate diagnosis of post traumatic radial artery pseudoaneurism in children. It is a primary important examination and exclude dangerous consecuences of excision in cases of misdiagnosis with an abscess. Early imaging with colour doppler ultrasonography is important for planning the definitive management. 


\section{International Journal of Science and Research (IJSR) \\ ISSN (Online): 2319-7064}

Index Copernicus Value (2013): 6.14 | Impact Factor (2014): 5.611

We strongly recommend dublex doppler ultrasound for evaluation of post traumatic mass swelling of extremities in children. Early imaging with dublex colour doppler ultrasound is important for planning the definitive management.

\section{References}

[1] Amrani, A., et al. "False aneurysm of the radial artery: Unusual complication of both-bone forearm fracture in children: A case report." Cases J 1 (2008): 170.

[2] Barker, C., P. Jefferson, and D. R. Ball. "Portable ultrasound to diagnose true radial artery aneurysm." Anesthesia \& Analgesia 105.3 (2007): 890-891.

[3] Bouch, D. C., and A. P. Hall. "Ultrasound diagnosis of a false radial artery aneurysm in ICU." Anaesthesia 61.10 (2006): 1018-1018.

[4] Canbaz, S., et al. "False aneurysm of the radial artery with sharp injury of the brachial artery, median and ulnar nerves: An unusual presentation."Journal of Cardiovascular Surgery 45.2 (2004): 169.

[5] Cozzi DA, Morini F, Casati A, et al. Radial artery pseudoaneurysm successfully treated by compression bandage. Arch Dis Child2003;88:165-6.

[6] Dzepina, Ivo, et al. "Pseudoaneurysms of the brachial artery following venipuncture in infants." Pediatric surgery international 20.8 (2004): 594-597.

[7] Gudena, R., and N. Khetan. "Swelling of volar aspect of the wrist."Postgraduate medical journal 81.958 (2005): e9.

[8] Herold, J., et al. "Ultrasound guided thrombin injection of pseudoaneurysm of the radial artery after percutaneous coronary intervention." Vasa 40.1 (2011): 78-81.

[9] Komorowska-Timek, Ewa, et al. "Treatment of radial and ulnar artery pseudoaneurysms using percutaneous thrombin injection." The Journal of hand surgery 29.5 (2004): 936-942.

[10] Milne, Charles PE, Ashok I. Handa, and Regent Lee. Iatrogenic Pseudoaneurysms. INTECH Open Access Publisher, 2012.

[11] Pelchovitz, Daniel J., et al. "Pseudoaneurysm in children: diagnosis and interventional management." Pediatric radiology 35.4 (2005): 434-439.

[12]Pero, Thomas, and John Herrick. "Pseudoaneurysm of the radial artery diagnosed by bedside ultrasound." Western Journal of Emergency Medicine10.2 (2009): 89.

[13] Poonai, Naveen, Rodrick Lim, and Tim Lynch. "Pseudoaneurysm formation following a traumatic wrist laceration." Cjem 13.01 (2011): 48-52.

[14]Pozniak, Myron A., Carol Mitchell, and Mike Ledwidge. "Radial Artery Pseudoaneurysm A Maneuver to Decrease the Risk of Thrombin Therapy."Journal of ultrasound in medicine 24.1 (2005): 119-122.

[15]Rozen, Galya, David R. Samuels, and Annat Blank. "The to and fro sign: the hallmark of pseudoaneurysm." The Israel Medical Association journal: IMAJ3.10 (2001): 781.

[16] Saad, Nael EA, et al. "Pseudoaneurysms and the Role of Minimally Invasive Techniques in Their Management 1." Radiographics 25.suppl_1 (2005): S173-S189.
[17] Saydam FA, Basaran K, Pilanci O, Ozel A. Posttraumatic pseudoaneurysm of the upper extremity in two pediatric patients. Hand Microsurg. 2013; 2(1): 35-38.

[18] Soto, Jorge A., et al. "Focal Arterial Injuries of the Proximal Extremities: Helical CT Arteriography as the Initial Method of Diagnosis 1." Radiology218.1 (2001): 188-194.

[19] Vlasta Žganjer, M. D., and M. D. Irenej Cigit. "Pseudoaneurysm of the brachial artery in a four-monthold boy: diagnosis and treatment." Archives of Iranian medicine 15.10 (2012): 650 .

[20] Wielenberg, A., et al. "Traumatic pseudoaneurysm of the brachial artery."Orthopedics 23.12 (2000): 12501322.

[21]Zitsman, Jeffrey L. "Pseudoaneurysm after penetrating trauma in children and adolescents." Journal of pediatric surgery 33.10 (1998): 1574-1577.

\section{Author Profile}



Dr. Besa Hidri has graduated in the Faculty of Medicine with excellent grades. She is professor at Faculty of the Medicine, University of Tirana. Currently works at Pediatric Imaging Service, Imaging Department, University Hospital Center "Mother Teresa" Tirana, Albania



Dr Durim Çela has graduated in the Faculty of Medicine with excellent grades. He is professor at Faculty of the Medicine, University of Tirana. Currently works at Imaging Service, Imaging Department, University Hospital Center "Mother Teresa" Tirana, Albania.

Dr Diamant Shtiza has graduated in the Faculty of Medicine with excellent grades. He is professor at Faculty of the Medicine, University of Tirana Currently works at Pediatric Department, University Hospital Center "Mother Teresa" Tirana, Albania. 